\title{
A Continuous Surjection from the Unit Interval onto the Unit Square
}

\author{
JARI TASKINEN
}

\begin{abstract}
We show that there exists a continuous surjection $\varphi: I \rightarrow I^{2}$ which admits an averaging operator in the sense of Pelczynski and which has the additional property that the map $\varphi^{a}: f \rightarrow f \circ \varphi$ is an isomorphism from $L_{p}\left(I^{2}\right)$ onto a subspace of $L_{p}(I)$, where $1 \leq p<\infty$.
\end{abstract}

\section{INTRODUCTION}

In [T] the author proved that for a wide class of pairs of compact metric spaces $\left(K, K_{1}\right)$ there exists a continuous surjection $\psi: K \rightarrow K_{1}$ admitting an averaging operator in the sense of Pełczyniski, [P]. The results of $[\mathrm{T}]$ contain the important special case that there exists a continuous surjection $\varphi: I \rightarrow I^{2}$, where $I=[0,1] \subset \mathbf{R}$, having a regular averaging operator (for the terminology, see below). The aim of this paper is to show that the definition of $\varphi$ can be modified such that $\varphi^{\circ}: f \rightarrow f \circ \varphi$ in addition becomes an isomorphism from $L_{p}\left(I^{2}\right)$ onto a subspace of $L_{p}(I)$, where $1 \leq p<\infty$ (Corollary 7 and Theorem 8). So, we get an operator $\varphi^{\circ}: C\left(I^{2}\right) \rightarrow C(I)$ which has good properties simultaneously with respect to the sup- and $L_{p}$-norms. This result, while being of interest in itself, is connected with the study of some Fréchet function spaces, see Section 4.

1991 Mathematics Subject Classification: primary 46B20, secondary 54C05

Editorial Complutense. Madrid, 1993. 
We introduce the notations and definitions used in this paper. If $K$ is a compact metric space, we denote by $C(K)$ the Banach space of continuous, real or complex valued mappings, endowed with the supnorm. If $K_{1}$ and $K_{2}$ are compact metric spaces and $\varphi: K_{1} \rightarrow K_{2}$ is a continuous surjection, we denote by $\varphi^{\circ}$ the linear isometry from $C\left(K_{2}\right)$ into $C\left(K_{1}\right)$ given by $\varphi^{\circ} f=f \circ \varphi$. If $\varphi^{\circ}\left(C\left(K_{2}\right)\right)$ is 1-complemented in $C\left(K_{1}\right)$, i.e., if there exists a contractive projection from $C\left(K_{1}\right)$ onto $\varphi^{\circ}\left(C\left(K_{2}\right)\right)$, we say that $\varphi$ admits a regular averaging operator. For more details we recommend the reference [LT], Sections II.4.h.i; see also [P].

Let $\Delta \subset I$ be the "middle thirds"-Cantor set; see for example [R], $\mathrm{p}$. 179. Using the homeomorphism

$$
\left(\varepsilon_{m}\right)_{m=1}^{\infty} \rightarrow \sum_{m=1}^{\infty} 2 \varepsilon_{m} 3^{-m}
$$

where $\varepsilon_{m}=0$ or 1 for all $m \in \mathbb{N}$, we identify the topological product

$$
\prod_{m=1}^{\infty}\{0,1\}
$$

with $\Delta$. By $\psi: \Delta \rightarrow[0,1]$ we denote the continuous surjection

$$
\psi\left(\left(\varepsilon_{m}\right)_{m=1}^{\infty}\right)=\sum_{m=1}^{\infty} \varepsilon_{m} 2^{-m}
$$

Each dyadic point of the form

$$
\sum_{m=0}^{n} \varepsilon_{m} 2^{-m} \in I
$$

where $\varepsilon_{n}=1, n \geq 1$, has two inverse images, $\left(\varepsilon_{1}, \ldots, \varepsilon_{n}, 0,0,0, \ldots\right)$ and $\left(\varepsilon_{1}, \ldots, \varepsilon_{n-1}, 0,1,1,1, \ldots\right)$. The other points of $I$ have only one inverse image. We define the discontinuous right inverse $\varrho: I \rightarrow \Delta$ of $\psi$ by

$$
\mathrm{Q}(x)=\min \{y \in \Delta \mid \psi(y)=x\},
$$

where "min" is taken with respect to the usual order of $I \supset \Delta$. The 
mapping $\mathrm{Q}^{\circ}$ is an isometry from $C(\Delta)$ onto

$D(I)$,

which is the subspace of $l_{\infty}(I)$ (the Banach space of bounded scalar valued functions on $I$ endowed with the sup-norm) spanned by continuous functions and the characteristic functions of intervals with dyadic endpoints. It is easy to check that such characteristic functions are contained in $\varrho^{\circ}(C(\Delta))$, and that the other details of this statement also hold.

The elements of $\Delta^{4}$ are considered as $4 \times \infty$-matrices consisting of numbers 0 or 1 (see (1.2)). We denote $4 \times 1$-matrices, i.e., the columns of elements of $\Delta^{4}$, by $\left(\varepsilon_{1}, \varepsilon_{2}, \varepsilon_{3}, \varepsilon_{4}\right)^{r}$. By $\overline{0}$ (resp. $\overline{1}$ ) we denote a matrix which consists of numbers 0 (resp. 1) only; the dimension of such a matrix will be clear from context. If $A=\left(\varepsilon_{i j}\right)$ is a matrix with $\varepsilon_{i j}=0$ or 1 for all $i$ and $j$, we denote by $A^{-}$the matrix $\left(\varepsilon_{i j}^{-}\right)$, where

$$
\varepsilon_{i j}^{-}= \begin{cases}0, & \text { if } \varepsilon_{i j}=1 \\ 1, & \text { if } \varepsilon_{i j}=0 .\end{cases}
$$

The space of $4 \times m$-matrices, consisting of numbers 0 and 1 , is denoted by $\Delta_{m}^{4}$.

We denote by $m_{n}$ the $n$-dimensional Lebesgue measure. We define the $\sigma$-algebra $\mathcal{M}$ of subsets of $\Delta$ by

$$
\mathcal{M}=\left\{\psi^{-1}(\mathcal{A}) \mid \mathscr{A} \subset I \text { is Lebesgue measurable }\right\},
$$

and we define the measure $\mu_{1}$ on $(\Delta, \mathcal{M})$ by $\mu_{1}(\mathcal{A})=m_{1}(\psi(\mathscr{A}))$, where $\mathcal{A} \in \mathcal{M}$. Note that $\mu_{1}$ is additive and even $\sigma$-additive in spite of the fact that $\psi$ is not an injection: if $\mathcal{A} \subset \Delta$ and $\mathcal{B} \subset \Delta$ are disjoint, then $\psi(\mathcal{A}) \cap \psi(\mathcal{B})$ is contained in the subset of the dyadic points of $I$; this set has Lebesgue measure 0 . We denote by $\mu_{n}$ the $n$-fold product of the measure $\mu_{1}$.

We define the homeomorphism $\eta: \Delta \rightarrow \Delta^{4}$, 


$$
\eta:\left(\varepsilon_{m}\right)_{m=1}^{\infty} \rightarrow\left(\left(\varepsilon_{4 m-3}\right)_{m=1}^{\infty},\left(\varepsilon_{4 m-2}\right)_{m=1}^{\infty},\left(\varepsilon_{4 m-1}\right)_{m=1}^{\infty},\left(\varepsilon_{4 m}\right)_{m=1}^{\infty}\right)^{T}
$$

By

$$
\sigma: I \times I \rightarrow I
$$

we mean the continuous surjection which assigns to $(x, y) \in I^{2}$ the unique number $t \in I$ such that $(x, y)$ belongs to the line segment joining $(0, t)$ with $\left(1, t^{2}\right)$. This map is used in the proof of the so called Milutin's lemma, see [LT], II.4.21.

\section{CONSTRUCTION OF THE MAP $\varphi$}

We first define the continuous surjection $\gamma: \Delta^{4} \rightarrow \Delta^{4}$ as follows.

Let $A \in \Delta^{4}$; we write $A=\left(A_{1}, A_{2}, A_{3}, \ldots\right)$ where each $A_{m}=\left(B_{m}, C_{m}\right)$ is a $4 \times 2$-matrix consisting of $(2 m-1)$ :th and $2 m$ :th columns $B_{m}$ and $C_{m}$ of $A$. We first define for all $m \in \mathbb{N}$ the $4 \times 1$-matrices $D_{m}$ inductively as follows. Let $D_{1}=C_{1}$. Let $m \in \mathbb{N}, m>1$, and assume that $D_{k}$ is defined for $k<m$. We first define $\Gamma_{A}^{m}:\{0,1\} \rightarrow \Delta_{l}^{4}$ by

$$
\begin{gathered}
\Gamma_{A}^{m}(0)= \begin{cases}D_{m-1}, & \text { if } A_{m-1}=\overline{0} \\
D_{m-1}^{-}, & \text {if } A_{m-1} \neq \overline{0},\end{cases} \\
\Gamma_{A}^{m}(1)= \begin{cases}D_{m-1}, & \text { if } A_{m-1}=\overline{1} \\
D_{m-1}^{-}, & \text {if } A_{m-1} \neq \overline{1}\end{cases}
\end{gathered}
$$

To define $D_{m}$ we distinguish between several cases.

1‥ If $B_{m} \neq C_{m}$ and $B_{m} \neq C_{m}^{-}$, we set $D_{m}=C_{m}$.

$2^{\circ}$. Assume that $A_{m}=\overline{0}$ or $\overline{1}$. If $A_{m}=A_{m-1}$, we set $D_{m}=D_{m-1}$, and if $A_{m} \neq A_{m-1}$, we set $D_{m}=D_{m-1}^{-}$. Remark. If $A_{m}=\overline{0}$, we have $D_{m}=\Gamma_{A}^{m}(0)$, and if $A_{m}=\overline{1}$, we have $D_{m}=\Gamma_{A}^{w_{1}}(1)$. 
3\%. a) Assume that $B_{m}=C_{m}, A_{m} \neq \overline{0}$ and $A_{m} \neq \overline{1}$. If $\Gamma_{A}^{m}(0)=C_{m}$, we set $D_{m}=(0,0,0,0)^{T}$. If $\Gamma_{A}^{m}(0) \neq C_{m}$, we set $D_{m}=C_{m}$.

b) Assume $B_{m}=C_{m}^{-}, C_{m} \neq(0,0,0,0)^{T}$ and $C_{m} \neq(1,1,1,1)^{T}$. If $\Gamma_{A}^{m}(1)=$ $C_{m}$, we set $D_{m}=(1,1,1,1)^{T}$ and $\Gamma_{A}^{m}(1) \neq C_{m}$, we define $D_{m}=C_{m}$.

$4^{\mathrm{o}}$. Consider the case $B_{m}=C_{m}^{*}$, and $C_{m}=(0,0,0,0)^{T}$ or $C_{m}=(1,1,1,1)^{T}$.

a) If $\Gamma_{A}^{m}(0)=(1,1,1,1)^{T}$ and $\Gamma_{A}^{m}(1) \neq(0,0,0,0)^{T}$, we set $D_{m}=(0,0,0,0)^{T}$.

b) If $\Gamma_{A}^{m}(1)=(0,0,0,0)^{T}$ and $\Gamma_{A}^{m}(0) \neq(1,1,1,1)^{T}$, we define $D_{m}=$ $(1,1,1,1)^{T}$.

c) In the other cases we set $D_{m}=C_{m}$.

We define the element $D \in \Delta^{4}$ by $D=\left(D_{1}, D_{2}, D_{3}, \ldots\right)$. We define also for all $m$ the mapping $\gamma_{m}: \Delta_{2 m}^{4} \rightarrow \Delta_{m}^{4}$ by

$$
\gamma_{m}\left(\left(A_{1}, \ldots, A_{m}\right)\right)=\left(D_{1}, \ldots, D_{m}\right)
$$

and we set

$$
\gamma(A)=D
$$
$I^{4} \rightarrow I^{2}$

In the following we denote $\bar{\psi}:=(\psi, \psi, \psi, \psi): \Delta^{4} \rightarrow I^{4}$ and $\bar{\sigma}:=(\sigma, \sigma)$ :

Lemma 1. The map $\gamma: \Delta^{4} \rightarrow \Delta^{4}$ is a continuous surjection which admits a continuous right inverse and for which the map

$$
\varphi=\bar{\sigma} \circ \bar{\psi} \circ \gamma^{\circ} \eta{ }^{\circ} \mathrm{Q}: I \rightarrow I^{2}
$$

is a continuous surjection.

Proof. The continuity of $\gamma$, the existence of a continuous right inverse of $\gamma$ and the surjectivity of $\varphi$ can be proved exactly as in Lemma 3.2 of [T]. Also the idea for the proof of the continuity of $\varphi$ is the same as in $[\mathrm{T}]$, but because of the details it is necessary to give the proof here. In 
view of Lemma 3.1 of [T] it is enough to show that

$$
J:=\bar{\psi} \circ \gamma \circ \eta: \Delta \rightarrow I^{4}
$$

maps the elements, $a, \bar{a} \in \Delta$ of the form

$$
\begin{aligned}
& a=(b, 1,0,0,0, \ldots) \\
& \tilde{a}=(b, 0,1,1,1, \ldots)
\end{aligned}
$$

where $b$ is a finite sequence consisting of numbers 0 or 1 , to the same element of $I^{4}$.

We denote $\eta(a)=A=\left(A_{1}, A_{2}, \therefore\right) \in \Delta^{4}$ and $\eta(\tilde{a})=\left(\tilde{A}_{1}, \tilde{A}_{2}, \ldots\right) \in \Delta^{4}$, where $A_{m}$ (respectively, $\bar{A}_{m}$ ) consists of the $2 m-1$; th and $2 m:$ th columns $B_{m}$ and $C_{m}$ of $A$ (resp. $\bar{B}_{m}, \bar{C}_{m}, \bar{A}$ ). In view of (10) and the definition of $\eta,(6)$, there exists a unique number $m$ such that $A_{k}=\tilde{A}_{k}$ for $k<m$ (if $m>1$ ), $A_{m} \neq \tilde{A}_{m}$ and $A_{k}=\overline{0}, \tilde{A}_{k}=\overline{1}$ for $k>m$.

We now consider $\gamma(A)=D=\left(D_{1}, D_{2}, \ldots\right)$ and $\gamma(\tilde{A})=\tilde{D}=\left(\tilde{D}_{1}, \tilde{D}_{2}, \ldots\right)$.

It is clear from the definition of $\gamma$ that $D_{k}=\bar{D}_{k}$ for $k<m$. Moreover, the matrix $A_{m}$ contains an element equal to 1 and, similarly, $\tilde{A}_{m}$ contains an element 0 . Hence, we have $A_{m} \neq A_{m+1}$ and $\tilde{A}_{m} \neq \tilde{A}_{m+1}$. By $2^{\mathrm{g}}$ we get $D_{m+1}=D_{m}^{-}$and $\tilde{D}_{m+1}=\tilde{D}_{m}^{-}$, and, moreover, $D_{k}=D_{m+1}$ and $\tilde{D}_{k}=\tilde{D}_{m+1}$ for $k>m+1$. So, we have

$$
\begin{aligned}
& \gamma \circ \eta(a)=D=\left(D_{1}, D_{2}, \ldots, D_{m-1}, D_{m}, D_{m}^{-}, D_{m}^{-}, D_{m}^{-}, \ldots\right), \\
& \gamma \circ \eta(\tilde{a})=\bar{D}=\left(D_{1}, D_{2}, \ldots, D_{m-1}^{\prime}, \tilde{D}_{m}, \tilde{D}_{m}^{-}, \tilde{D}_{m}^{-}, \tilde{D}_{m}^{-}, \ldots\right) .
\end{aligned}
$$

Let us consider the $i$ :th $(1 \leq i \leq 4)$ rows $\left(d_{1}^{(i)}, d_{2}^{(i)}, \ldots\right) \in \Delta$ and $\left(\bar{d}_{1}^{(i)}, \bar{d}_{2}^{(i)}, \ldots\right)$ $\in \Delta$ of $D$ and $\tilde{D}$, respectively. We have $d_{j}^{(i)}=\bar{d}_{j}^{(i)}$ for $1 \leq j<m$. Moreover, $d_{\mathrm{k}}^{(i)}=d_{\mathrm{m}}^{(i)}$ and $\tilde{d}_{\mathrm{k}}^{(i)}=\tilde{d}_{\mathrm{m}}^{(i)-}$ for all $k>m$. This shows that

$$
\psi\left(\left(d_{1}^{(i)}, d_{2}^{(i)}, \ldots\right)\right)=\psi\left(\left(\tilde{d}_{1}^{(i)}, \tilde{d}_{2}^{(i)}, \ldots\right)\right)
$$

and, hence, $J(a)=\bar{\psi}(D)=\bar{\psi}(\bar{D})=J(\tilde{a})$. 
Theorem 2. The map $\varphi: l \rightarrow I^{2}$ (see (8)) has a regular averaging operator.

The proof is the same as that of Theorem 3.3 of [T].

\section{MAIN RESULT}

We now show that $\varphi$ also has the additional property that $\varphi^{\circ}$ defines an isomorphism from $L_{p}\left(I^{2}\right)$ into $L_{p}(I)$ for $1 \leq p<\infty$.

Lemma 3. Let $m>I$ and $A \in \Delta_{2 m \cdot 2}^{4}$ and $D \in \Delta_{l}^{4}$. There exist exactly 16 different matrices $A_{m} \in \Delta_{2}^{4}$ such that

$$
\gamma_{m}\left(\left(A, A_{m}\right)\right)=\left(\gamma_{m-1}(A), D\right) \text {. }
$$

Proof. The proof of this lemma consists of a straightforward but elaborate verification of the different cases in the definition of $\gamma_{m}$. The numbers $1^{\circ}-4^{\circ}$ refer there.

i) We first assume that $D \neq(0,0,0,0)^{T}$ and $D \neq(1,1,1,1)^{T}$. There exist 14 vectors $B \in \Delta_{1}^{4}$ such that $B \neq D$ and $B \neq D$. By $1^{10}$ we see that $A_{m}=$ $(B, D)$ satisfies (11) for all such $B$.

Next we check if the cases $A_{m}=\overline{0}, \overline{1},(D, D)$ or $\left(D^{-}, D\right)$ could satisfy (11). First, if $\Gamma_{A}^{m}(0)=D$, then, by $2^{\circ}$ and $\left.3^{\circ} \mathrm{a}\right), A_{m}=\overline{0}$ satisfies (11) and $A_{m}=(D, D)$ does not (use the remark in $2^{\mathrm{g}}$ ). If $\Gamma_{A}^{m}(0) \neq D$, then $A_{m}=(D, D)$ satisfies (11) and $A_{m}=\overline{0}$ does not. Hence, in every case exactly one of the matrices $\overline{0}$ and $(D, D)$ satisfies (11). In the same way we see that exactly one the matrices $\overline{1}$ and $\left(D^{\circ}, D\right)$ satisfies (11).

Since $D \neq(0,0,0,0)^{T},(1,1,1,1)^{T}$ we see that $4^{\text {Q }}$ cannot produce other matrices satisfying (11). Finally, by $1^{9}-4^{\circ}$, a matrix $A_{m}$ of the form $A_{m}=$ $(B, C)$, where $C \neq D$ and, moreover, either $B$ or $C$ is different from $(0,0,0,0)^{T}$ and $(1,1,1,1)^{T}$, cannot satisfy $(11)$. 
Summing up, we see that (11) holds for exactly 16 different matrices $A \in \Delta_{2}^{4}$.

ii) We assume $D=(0,0,0,0)^{T}$. Again there exist 14 vectors $B \in \Delta_{1}^{4}$, $B \neq D$ and $B \neq D^{-}$. By $1^{\circ}$, (11) holds for $A_{m}=(B, D)$.

It follows immediately from $1 \%-4^{\circ}$ that a matrix $A_{m}=\left(B_{m}, C_{m}\right)$, where $C_{m} \neq D$, can satisfy (11) only if $C_{m}=D^{-}$and $B_{m}=D$ or $D$, or if $B_{m}=C_{m}$ $=\Gamma_{A}^{m}(0)$ (see $3^{o}$ a)). Hence, we need only to consider such cases and the cases $A_{m}=\overline{0}$ and $A_{m}=\left(D^{-}, D\right)$. We should find exactly two matrices of these types satisfying (11).

a) We assume $\Gamma_{A}^{m}(0)=(0,0,0,0)^{T}$. Then $A_{m}=\overline{0}$ satisfies (11). Moreover, if $\Gamma_{A}^{m}(1)=(0,0,0,0)^{T}$, then also $A_{m}=\overline{1}^{m}$ works, by $2^{\alpha}$, and, by $\left.4^{\circ} \mathrm{b}\right)$, the cases $A_{m}=\left(D^{-}, D\right), A_{m}=\left(D, D^{\prime}\right)$ do not work. If $\Gamma_{A}^{m}(1) \neq$ $(0,0,0,0)^{T}$, then $A_{m}=\overline{1}$ (see $2^{\circ}$ ) and $A_{m}=\left(D, D^{-}\right)$(see $4^{\circ}$ c)) do not work but $A_{m}=\left(D^{-}, D\right)$ does, by $\left.4^{\circ} \mathrm{c}\right)$. So we get altogether two positive cases.

b) We assume $\Gamma_{A}^{m}(0)=(1,1,1,1)^{T}$ so that $A_{m}=\overline{0}$ does not work. If $\Gamma_{A}^{m}(1)=(0,0,0,0)^{T}$, then by $2^{\circ}, A_{m}=\overline{1}$ satisfies (11). Moreover, by $4^{\circ} \mathrm{c}$ ), $\left(D^{\top}, D\right)$ satisfies $(11)$ and $\left(D, D^{-}\right)$does not. If $\Gamma_{A}^{m}(1) \neq(0,0,0,0)^{T}$, then $A_{m}=$ $\overline{1}$ does not satisfy $(11)$, but by $\left.4^{\mathrm{o}} \mathrm{a}\right),\left(D^{-}, D\right)$ and $\left(D, D^{-}\right)$do.

c) Assume $\Gamma_{A}^{m}(0) \neq D$ and $\Gamma_{A}^{m}(0) \neq D^{-}$. The case $A_{m}=\overline{0}$ does not work. By $3^{\underline{o}}$ a), $A_{m}=\left(\Gamma_{A}^{m}(0), \Gamma_{A}^{m}(0)\right)$ satisfies (11). If $\Gamma_{A}^{m}(1)=(0,0,0,0)^{T}$, then $A_{m}=\overline{1}$ works, and by $4^{\circ}$ b), $A_{m}=\left(D, D^{-}\right)$and $A_{m}=\left(D^{-}, D\right)$ do not. If $\Gamma_{A}^{m}(1) \neq(0,0,0,0)^{T}$, then $A_{m t}=\overline{1}$ does not work, and by $\left.4^{\mathrm{g}} \mathrm{c}\right), A_{m}=\left(D^{-}, D\right)$ satisfies (11) and $A_{m}=\left(D, D^{-}\right)$does not.

iii) The case $D=(1,1,1,1)^{T}$ is analogous to ii). But since the point in this kind of proofs is a careful verification of all the details, we want to give the proof also in this case.

By $1^{\circ}$, there exist 14 vectors $B \in \Delta_{1}^{4}, B \neq D, B \neq D^{-}$such that (11) holds for $A_{m}=(B, D)$. From now on we need only to consider the cases $A_{m}=\overline{1}, \overline{0},\left(D^{-}, D\right),\left(D, D^{-}\right)$and $\left(\Gamma_{A}^{m}(1)^{-}, \Gamma_{A}^{m}(1)\right)$. 
a) We assume $\Gamma_{A}^{m}(1)=D$. Now $A_{m}=\overline{1}$ works. If $\Gamma_{A}^{m}(0)=D$, then also $\overrightarrow{0}$ works but $\left(D^{*}, D\right)$ and $\left(D, D^{-}\right)$do not. If $\Gamma_{A}^{m}(0) \neq D$, then $\overline{0}$ and $\left(D, D^{\circ}\right)$ do not work but $\left(D^{-}, D\right)$ does.


but $\overline{1}$ and $\left(D, D^{-}\right)$do not. If $\Gamma_{A}^{m}(0) \neq D$, then the cases $A_{m}=\overline{0}$ and $A_{m}=\overline{1}$ are negative and the cases $\left(D^{-}, D\right)$ and $\left(D, D^{-}\right)$are positive.

c) Assume $\Gamma_{A}^{m}(1) \neq D, D^{-}$. By $3^{0}$ b), $A_{m}=\left(\Gamma_{A}^{m}(1)^{-}, \Gamma_{A}^{m}(1)\right)$ satisfies (11). If $\Gamma_{A}^{m}(0)=D$, then also $\overline{0}$ works but $\overline{1},\left(D^{-}, D\right)$ and $\left(D, D^{-}\right)$do not. If $\Gamma_{A}^{m}(0)$ $\neq D$, then $\overline{0}, \overline{1}$ and $\left(D, D^{-}\right)$do not work but $\left(D^{-}, D\right)$ does.

We have now gone through all the cases.

Corollary 4. Given $m \in N$ and $D \in \Delta_{m}^{4}$ there exist exactly $2^{4 m}$ different matrices $A \in \Delta_{2 m}^{4}$ such that $\gamma_{m}(A)=D$.

Proof. Let $A=\left(A_{1}, \ldots, A_{m}\right) \in \Delta_{2 m}^{4}$, where $A_{m} \in \Delta_{2}^{4}$, and let $A^{*}=$ $\left(A_{1}, \ldots, A_{m-1}\right)$. Since the matrix formed by the first $m-1$ columns of $\gamma_{m}(A)$ is equal to $\gamma_{m-1}\left(A^{\prime}\right)$, we can prove Corollary 4 using Lemma 3 and induction with respect to the number of the columns of $D$. Note that by definition, the 16 matrices $(B, C)$, where $B \in \Delta_{1}^{4}$, are the preimages of $C$ with respect to $\gamma_{1}$.

Lemma 5. Let $K(i) \in \mathbb{N}$ for all $i=1,2,3,4$ and let $a_{m}^{(i)} \in\{0,1\}$ for all $i$ and for all $m \leq K(i)$. Let us denote by $A \subset \Delta^{4}$ the set

$$
A=\left\{\left(x_{m}^{(i)}\right)_{\substack{m \in \mathbb{N} \\ \text { sust }}} \in \Delta^{4} \mid x_{m}^{(i)}=a_{m}^{(i)} \text { for } m \leq K(i)\right\}
$$

We have

$$
\mu_{4}\left(\gamma^{-1}(A)\right)=\prod_{i=1}^{4} 2^{-K(0)}
$$


Proof. Let $K=\max \{K(i) \mid 1 \leq i \leq 4\}$, Let us introduce the set ,

$$
\mathcal{A}_{K}=\left\{\left(x_{m}^{(i)}\right)_{\substack{1 \leq m \leq K \\ 1 \leq \leq \leq 4}} \in \Delta_{K}^{4} \mid x_{m}^{(i)}=a_{m}^{(i)} \text { for } m \leq K(i)\right\} .
$$

It is a direct consequence of the definition of $\gamma$ and $\gamma_{K}$ that $A \in \gamma^{\prime}(\mathcal{A})$ if and only if $A=\left(A_{1}, B\right)$, where $A_{1} \in \Delta_{2 K}^{4}, B \in \Delta^{4}$ and $A_{1}$ satisfies

$$
\gamma_{K}\left(A_{1}\right) \in \mathcal{A}_{K}
$$

For a fixed $A_{1} \in \gamma_{K}^{-1}\left(A_{K}\right) \subset \Delta_{2 K}^{4}$

$$
\mu_{4}\left(\left(\left(A_{1}, B\right) \in \Delta^{4} \mid B \in \Delta^{4}\right\}\right)=2^{-8 K} .
$$

We thus need only to calculate $\#\left(\gamma_{K}^{-1}\left(\mathcal{A}_{K}\right)\right)$. (We denote by $\#(C)$ the cardinality of the set $C$ ). But by Corollary 4 ,

$$
\#\left(\gamma_{K}^{-1}\left(\mathcal{A}_{K}\right)\right)=2^{4 K} \#\left(\mathcal{A}_{K}\right)
$$

On the other hand it is elementary to see that

$$
\#\left(\mathcal{A}_{K}\right)=\prod_{i=1}^{4} 2^{K-K(1)} .
$$

Since the sets $\left\{(A, B) \mid B \in \Delta^{4}\right\}$ and $\left\{\left(A_{1}, B\right) \mid B \in \Delta^{4}\right\}$ are disjoint for $A \neq$ $A_{1}$, we get by (15) and (16)

$$
\mu_{A}\left(\gamma^{-1}(\mathcal{A})\right)=2^{-8 K} \#\left(\gamma_{K}^{-1}\left(\mathcal{A}_{K}\right)\right)=2^{-4 K} \prod_{i=1}^{4} 2^{K-K(i)}=\prod_{i=1}^{4} 2^{-K(i)} .
$$

Proposition 6. There exist positive constants $C_{1}$ and $C_{2}$ such that

$$
C_{1} m_{1}\left(\varphi^{-1}(\mathcal{A})\right) \leq m_{2}(\mathcal{A}) \leq C_{2} m_{1}\left(\varphi^{-1}(\mathcal{A})\right)
$$

holds for all rectangles $\mathcal{A} \subset I^{2}$. 
Proof. 19. We first find positive constants $d_{1}$ and $d_{2}$ such that

$$
d_{1} \mu_{4}\left(\gamma^{-1}(\mathcal{A})\right) \leq \mu_{4}(\mathcal{A}) \leq d_{2} \mu_{4}\left(\gamma^{-1}(\mathcal{A})\right)
$$

holds for all rectangles $A \subset \Delta^{4}$,

$$
\mathcal{A}=\prod_{i=1}^{4}\left[a^{(i)}, \tilde{a}^{(i)}\right]
$$

where $a^{(i)}, \tilde{a}^{(i)} \in \Delta$ for all $i$. (Intervals $\left[a^{(i)}, \tilde{a}^{(i)}\right]$ in $\Delta$ are defined with respect to the natural order of $\Delta$.)

Let us denote for $x=\left(x_{m}\right)_{m=1}^{\infty}, y=\left(y_{m}\right)_{m=1}^{\infty} \in \Delta, x \neq y$,

$$
n(x, y)=\min \left\{m \mid x_{m} \neq y_{m}\right\}
$$

and let $m(a, i)$ (resp. $m(\tilde{a}, i))$ stand for the smallest number $m$ such that $m$ $>n\left(a^{(i)}, \tilde{a}^{(i)}\right)$ and $a_{m}^{(i)}=0$ (resp. $\tilde{a}_{m}^{(i)}=1$ ). Then $\left(^{*}\right) x \in\left[a^{(i)},,^{(i)}\right]$ if either $x_{m}$ $=a_{m}^{(i)}$ for $m<m(a, i)$ and $x_{m(a, i)}=1$, or $x_{m}=\tilde{a}_{m}^{(i)}$ for $m<m(\tilde{a}, i)$ and $x_{m(\tilde{a}, i)}$ $=0$. Moreover, $\left({ }^{* *}\right)$ if $x \in\left[a^{(i)}, \tilde{a}^{(i)}\right]$ then either $x_{m}=a_{m}^{(i)}$ for $m<m(a, i)$, or $x_{m}=\tilde{a}_{m}^{(j)}$ for $m<m(\tilde{a}, i)$. Denoting $M(i):=\min \{m(a, i), m(\tilde{a}, i)\}$ for all $1 \leq i \leq 4$ we thus get

$$
2^{-M(i)} \leq \mu_{1}([a, \vec{a}]) \leq 2^{-M(i)+2}
$$

so that,

$$
\prod_{i=1}^{4} 2^{-M(i)} \leq \mu_{4}(\mathcal{A}) \leq 2^{8} \prod_{i=1}^{4} 2^{-M(i)}
$$

Let us denote, for all $i, b_{m}^{(i)}=a_{m}^{(i)}$ and $b_{M(i)}=1$, if $M(i)=m(a, i)$, or $b_{m}^{(i)}=\bar{a}_{m}^{(i)}$ and $b_{M(i)}=0$, if $M(i)=m(\bar{a}, i)$. From $\left(^{*}\right)$ we see that $\mathcal{A}$ contains the set

$$
\mathcal{B}=\left\{\left(x_{m}^{(i)}\right) \in \Delta^{4} \mid x_{m}^{(i)}=b_{m}^{(i)} \text { for } m \leq M(i)\right\}
$$


By Lemma 5,

$$
\mu_{4}\left(\gamma^{-1}(\mathcal{A})\right) \geq \mu_{4}\left(\gamma^{-1}(\mathcal{B})\right)=\prod_{i=1}^{4} 2^{-M(i)}
$$

Moreover, by (**) $A$ is contained in the union of the 16 sets

$$
\mathcal{B}\left(\varepsilon_{1}, \varepsilon_{2}, \varepsilon_{3}, \varepsilon_{4}\right)=\left\{\left(x_{m}^{(i)}\right) \in \Delta^{4} \mid x_{m}^{(i)}=c_{m}^{(i)} \text { for } m<M(i)\right\}
$$

where $\varepsilon_{i} \in\{0,1\}$ for $i=1, \ldots, 4$ and $c_{m}^{(i)}=a_{m}^{(i)}$ for $m<M(i)$, if $\varepsilon_{i}=0$, or $c_{m}^{(i)}=\tilde{a}_{m}^{(i)}$ for $m<M(i)$, if $\varepsilon_{i}=1$. Hence, by Lemma 5

$$
\mu_{4}\left(\gamma^{-1}(\mathcal{A})\right) \leq \mu_{4}\left(\bigcup_{\varepsilon_{i} \in[0,1\}} \gamma^{-1}\left(B\left(\varepsilon_{1}, \varepsilon_{2}, \varepsilon_{3}, \varepsilon_{4}\right)\right)\right) \leq 2^{8} \prod_{i=1}^{4} 2^{-M(i)}
$$

Combining (20), (22) and (24) we see that (17) holds with $d_{1}=2^{-8}$, $d_{2}=2^{8}$.

$2^{\mathrm{o}}$. We find constants $c_{1}, c_{2}>0$ such that

$$
c_{1} \mu_{1}\left(\eta^{-1}(\mathscr{A})\right) \leq \mu_{4}(\mathscr{A}) \leq c_{2} \mu_{1}\left(\eta^{-1}(\mathscr{A})\right)
$$

holds for all 4-rectangles $\mathcal{A} \subset \Delta^{4}$. We define the elements $a^{(i)}$ and $\tilde{a}^{(i)}$ and the numbers $m(a, i), m(\tilde{a}, i), b_{t t}^{(i)}$ and $M(i)$ as in $1^{\mathbf{0}}$. Let $M=\max |M(i)|$ $i=1,+, 4\}$. Note that by $(20)$ we again have

$$
\prod_{i=1}^{4} 2^{-M(i)} \leq \mu_{4}(\mathcal{A}) \leq 2^{8} \prod_{i=1}^{4} 2^{-M(i)}
$$

Let us define the set $\mathcal{B} \subset \mathcal{A}$ as in $1^{\circ}$, (21). From the definition of $\eta$, (6), we see that

$\eta^{-1}(\mathcal{B})=\left\{\left(x_{m}\right)_{m=1}^{\infty} \in \Delta_{4 M} \mid x_{4(m-1)+i}=b_{m}^{(i)}\right.$ for all $1 \leq i \leq 4$ and $\left.m \leq M(i)\right\}$.

Let us denote 
$C=\left\{\left(x_{m}\right)_{m=1}^{4 M} \in \Delta_{4 M} \mid x_{4(m-1)+i}=b_{m}^{(i)}\right.$ for all $1 \leq i \leq 4$ and $\left.m \leq M(i)\right\}$.

Now $x=\left(x_{m}\right) \in \eta^{-1}(\mathcal{B})$ if and only if $x=(y, z)$, where $y \in C$ and $z \in \Delta$. For a fixed $y \in C$ we have

$$
\mu_{1}\left(\left((y, z) \mid z \in \Delta^{\prime}\right)=2^{-4 M} .\right.
$$

We calculate $\#(C)$. In view of (28), the elements of $C$ are vectors with $4 M$ components out of which $\Sigma_{i=1}^{4} M(i)$ are fixed and thus $4 M-\Sigma_{i=1}^{4} M(i)$ may be chosen arbitrarily from the set $\{0,1\}$. So,

$$
\#(C)=2^{4 M-\Sigma_{i-1} M(1)} .
$$

Combining this with (29) we get

$$
\mu_{1}\left(\eta^{-1}(\mathcal{A})\right) \geq \mu_{1}\left(\eta^{-1}(\mathcal{B})\right)=\prod_{i=1}^{4} 2^{-M(i)} .
$$

To get an upper estimate for $\mu\left(\eta^{-1}(\mathcal{A})\right)$ we define the 16 sets $\mathcal{B}\left(\varepsilon_{1}, \varepsilon_{2}, \varepsilon_{3}, \varepsilon_{4}\right)$, where $\varepsilon_{i} \in\{0,1\}$, as in $1^{\circ},(23)$. Since these sets are of the same form as $\mathcal{B}$ above, we get by (31)

$$
\mu_{1}\left(\eta^{-1}\left(\mathcal{B}\left(\varepsilon_{1}, \varepsilon_{2}, \varepsilon_{3}, \varepsilon_{4}\right)\right)\right)=\prod_{i=1}^{4} 2^{-M(3)+1}
$$

Since the union of all the sets $\mathcal{B}\left(\varepsilon_{1}, \varepsilon_{2}, \varepsilon_{3}, \varepsilon_{4}\right)$ contains $\mathcal{A}$, we get from (32)

$$
\mu_{1}\left(\eta^{-1}(\mathcal{A})\right) \leq 2^{8} \prod_{i=1}^{4} 2^{-M(i)} \text {. }
$$

Combining (26), (31) and (33) yields (25) with $c_{1}=2^{-8}, c_{2}=2^{8}$.

3o. We consider the map $\bar{\sigma}$. If $[a, b] \subset I$, the definition of $\sigma$ implies 


$$
m_{2}\left(\sigma^{-1}([a, b])\right)=\left(\left(b+b^{2}\right)-\left(a+a^{2}\right)\right) / 2=(b-a)(1+b+a) / 2 .
$$

Hence, $m_{1}([a, b]) / 2 \leq m_{2}\left(\sigma^{-1}([a, b]) \leq 2 m_{1}([a, b])\right.$, and so

$$
m_{4}\left(\bar{\sigma}^{-1}(\mathcal{A})\right) / 4 \leq m_{2}(\mathcal{A}) \leq 4 m_{4}\left(\bar{\sigma}^{-1}(\mathcal{A})\right)
$$

for all rectangles $A \subset I^{2}$.

$4^{\circ}$. Our statement now follows by combining (17), (25) and (34); the maps $\varrho$ and $\bar{\psi}$ are measure preserving. Note that if $\mathscr{A} \subset I^{2}$ is a rectangle, then $\sigma^{-1}(\mathcal{A})$ is not a 4-rectangle but there is no difficulty to approximate it as well as we wish by finite unions of 4-rectangles. Moreover, if $\mathcal{A} \subset \Delta^{4}$ is a rectangle, then $\gamma^{-1}(\mathcal{A})$ need not be. However, the lower and upper estimates for $\mu_{4}\left(\gamma^{-1}(\mathscr{A})\right)$ are done using 4-rectangles in $\Delta^{4}$, see (21) and (23), respectively. Hence, we need also the result of $2^{2}$ only for rectangles.

Corollary 7. There exist positive constants $C_{1}$ and $C_{2}$ such that for all $f \in C\left(I^{2}\right)$

$$
C_{1} \int_{I}|f \circ \varphi(x)| d x \leq \int_{I^{2}}|f(x)| d x \leq C_{2} \int_{I}|f \circ \varphi(x)| d x \text {. }
$$

Proof. If $\left(A_{i}\right)_{i=1}^{n}$ is a sequence of disjoint rectangles in $I^{2}$, we have for all sequences $\left(a_{i}\right)_{i=1}^{n}$ of scalars

$$
\begin{aligned}
& \int_{i^{2}}\left|\sum_{i=1}^{n} a_{i} \chi_{i}(x)\right| d x=\sum_{i=1}^{n}\left|a_{i}\right| m_{2}\left(A_{i}\right), \\
& \int_{i}\left|\sum_{i=1}^{n} a_{i} \chi_{i} \circ \varphi(x)\right| d x=\sum_{i=1}^{n}\left|a_{i}\right| m_{1}\left(\varphi^{-1}\left(A_{i}\right)\right),
\end{aligned}
$$

where $\chi_{i}$ is the characteristic function of $A_{i^{*}}$ So, for simple functions of 
this form (35) follows from Proposition 6, and for continuous functions we get the statement by approximation.

We immediately get the following

Theorem 8. The operator $\varphi^{\circ}$ can be extended to an isomorphism from $L_{p}\left(I^{2}\right)$ into $L_{p}(I)$, where $l \leq p<\infty$.

\section{ON THE SPACES $C(\Omega) \cap L_{p}(\Omega)$}

If $\Omega \subset \mathbf{R}^{n}, n \geq 1$, is an open set, we denote by $C(\Omega) \cap L_{p}(\Omega)$, $1 \leq p<\infty$, the Fréchet space of continuous, $L_{p}$-integrable functions from $\Omega$ into $\mathbb{K}$. The topology of this space is determined by the seminorms

$$
\begin{aligned}
& p_{0}(f)=\left(\int_{\Omega}|f|^{p}\right)^{1 / p}, \\
& p_{k}(f)=\sup _{x \in \Omega_{k}}|f(x)|, \quad k \in \mathbb{N}
\end{aligned}
$$

where $\left(\Omega_{k}\right)_{k=1}^{\infty}$ is an increasing sequence of compact subsets of $\Omega$, whose union is $\Omega$. For more detajls on these spaces we refer to [BT].

The isomorphic classification of such spaces is an open problem. Probably the most interesting question in this area is, whether the spaces $C(\mathbb{R}) \cap L_{p}(\mathbb{R})$ and $C\left(\mathbb{R}^{2}\right) \cap L_{p}\left(\mathbb{R}^{2}\right)$ are isomorphic to each other. It is not difficult to see, using a natural imbedding, that $C(\mathbf{R}) \cap L_{p}(\mathbb{R})$ is isomorphic to a complemented subspace of $C\left(\mathbb{R}^{2}\right) \cap L_{p}\left(\mathbb{R}^{2}\right)$. (First, select a continuous cut-off function with compact support $\varphi \in C(\mathbf{R})$ such that $0 \leq \varphi \leq 1$ on $\mathbb{R}$ and $\varphi=1$ for every $x \in[0,1]$. Put $E:=C\left(\mathbb{R}^{2}\right) \cap L_{p}\left(\mathbb{R}^{2}\right)$ and $F:=C(\mathbb{R}) \cap L_{p}(\mathbb{R})$ and define $T: F \rightarrow E$ by $T f(x, y)=f(x) \varphi(y)$ for all $f \in F$, $x, y \in \mathbb{R}$, and $S: E \rightarrow F$ by $S g(x):=\int_{0}^{1} g(x, y) d y$ for all $g \in E, x \in \mathbb{R}$. It is a direct matter to check that $T$ and $S$ are continuous linear maps such that $S \circ T$ is the identity of $F$. So, $P:=T \circ S$ is a continuous projection on $E$ whose image is isomorphic to $F$.) So, in view of the decomposition 
method of Pełczyński, the crucial problem is, whether $C\left(\mathbb{R}^{2}\right) \cap L_{p}\left(\mathbb{R}^{2}\right)$ is isomorphic to a complemented subspace of $C(\mathbb{R}) \cap L_{p}(\mathbb{R})$. Using the results in earlier sections we can prove that the space defined on $\mathbb{R}^{2}$ is isomorphic to a subspace of $C(\mathbb{R}) \cap L_{p}(\mathbb{R})$, but to prove the complementedness we would still need a "measure preserving" continuous surjection $\varphi_{:} I \rightarrow I^{2}$ and a projection $P$ which is simultaneuously bounded $C(I) \rightarrow \varphi^{\circ}\left(C\left(I^{2}\right)\right)$ and $L_{p}(I) \rightarrow \varphi^{\circ}\left(L_{p}\left(I^{2}\right)\right)$, and this result is not (yet?) available.

So, let us prove what we can do.

Lemma 9. There exists an enumeration $\left(Q_{n}\right)_{n \in Z}$ of the family of closed squares $\left(Q_{n, m}\right)_{n, m \in Z}$, where $Q_{n, m}=\left\{(x, y) \in \mathbb{R}^{2} \mid n \leq x \leq n+1, m \leq\right.$ $y \leq m+1]$, such that $Q_{n}$ and $Q_{n+1}$ have a common side for all $n \in Z$.

There is no difficulty to make such an enumeration for example according to the following picture:

\begin{tabular}{|c|c|c|c|c|c|c|c|c|}
\hline & -17 & -16 & 15 & 16 & 17 & 18 & \\
\hline-13 & -14 & -15 & 14 & 13 & 12 & 19 & - \\
\hline-12 & -3 & -2 & 1 & 2 & 11 & 20 & \\
\hline-11 & -4 & -1 & 0 & 3 & 10 & 21 \\
\hline-10 & -5 & -6 & 5 & 4 & 9 & 22 \\
\hline-9 & -8 & -7 & 6 & 7 & 8 & 23 \\
\hline & & & 27 & 26 & 25 & 24 \\
\hline
\end{tabular}

Figure 1.

Proposition 10. For all $p, I \leq p<\infty$, the space $C\left(\mathbb{R}^{2}\right) \cap L_{p}\left(\mathbb{R}^{2}\right)$ is isomorphic to a subspace of $C(\mathbf{R}) \cap L_{p}(\mathbb{R})$. 
Proof. Let $\varphi$ be the map constructed in Section 2. It can be verified from the definition that $\varphi(0)=(0,0)$ and $\varphi(1)=(1,1)$. Let $\psi^{(1)}$ be a homeomorphism from $I^{2}$ onto itself such that $\psi^{(1)}(0,0)=(0,0)$ and $\psi^{(1)}(1,1)$ $=(1,0)$. It is then clear that also the operator $\psi^{\circ}$, where $\psi:=\psi^{(1)} \circ \varphi$, is an isometry from $C\left(I^{2}\right)$ onto a subspace of $C(I)$ and an isomorphism from $L_{p}\left(I^{2}\right)$ onto a subspace of $L_{p}(I)$. Let $\left(Q_{n}\right)_{n=-\infty}^{\infty}$ be the sequence of closed squares as in Lemma 9. We claim that it is possible to choose a sequence of continuous surjections $\varphi^{(n)}:[n, n+1] \rightarrow Q_{n}$ such that

(i)) each $\varphi^{(n)}$ is of the form $\tau_{n}^{(2)} \circ r_{n} \circ \varphi \circ \tau_{n}$ or $\tau_{n}^{(2)} \circ r_{n} \circ \psi \circ \tau_{n}$, where $\tau_{n}$ is the translation from $[n, n+1]$ onto $l, r_{n}$ is an isometry from $l^{2}$ onto itself, and $\tau_{n}^{(2)}$ is the translation from $I^{2}$ onto $Q_{n}$, and

(ii)) $\varphi^{(n-1)}(n)=\varphi^{(n)}(n)$ for all $n \in Z$.

Note that ii) means in particular that

$$
\varphi^{(n-1)}(n) \in Q_{n^{*}}
$$

To prove this we first choose $\varphi^{(0)}$ of the form i) such that $\varphi^{(0)}(0) \in$ $Q_{\text {. }}$ and $\varphi^{(0)}(1) \in Q_{1}$. Assume that $n \geq 1$ and that $\varphi^{(k)}$ is constructed for $-n+1 \leq k \leq n-1$ such that i) holds for these $\varphi^{(k)}$ and such that $\varphi^{(k-1)}(k)=$ $\varphi^{(k)}(k)$ for $-n+1<k \leq n-1$ and $\varphi^{(n-1)}(n) \in Q_{n}$ and $\varphi^{(-n+1)}(-n+1) \in Q_{-n^{*}}$ By Lemma 9 the squares $Q_{n}$ and $Q_{n+1}$ have one common side $S_{n}$. So, it is possible to join one of the endpoints, say $S_{n}$, of $S_{n}$ and $\varphi^{(n-1)}(n)$ by one side of $Q_{n}$ or one diagonal of $Q_{n}$. We thus can find a map $\varphi^{(n)}$ which is of the form i) and satisfies $\varphi^{(n)}(n)=\varphi^{(n-1)}(n)$ and $\varphi^{(n)}(n+1)=s_{n} \in Q_{n+1}$. (If $s_{n}$ and $\varphi^{(n-1)}(n)$ are the endpoints of a side of $Q_{n}$, we can take a map of the form $\tau_{n}^{(2)} \circ r_{n} \circ \psi \circ \tau_{n}$, and if $s_{n}$ and $\varphi^{(n-1)}(n)$ are in the opposite corners of $Q_{n}$, i.e. they are the endpoints of a diagonal of $Q_{n}$, we can take a map of the form $\tau_{n}^{(2)} \circ r_{n} \circ \varphi \circ \tau_{n}$. In each case $r_{n}$ is the combination of some rotation and reflection.) The map $\varphi^{(-n)}$ is defined analogously.

Defining

$$
\phi(t)=\varphi^{(n)}(t) \text { for } t \in[n, n+1], n \in Z,
$$

we get a continuous surjection from $\mathbf{R}$ onto $\mathbf{R}^{2}$. We claim that $\phi^{\circ}: f \rightarrow f \circ \phi$ 
is the desired isomorphism from $C\left(\mathbb{R}^{2}\right) \cap L_{p}\left(\mathbf{R}^{2}\right)$ onto a subspace of $C(\mathbf{R})$ $\cap L_{p}(\mathbf{R})$. Since $\phi$ is a continuous surjection and since $\phi^{-1}(K)$ is compact for all compact $K \subset \mathbf{R}^{2}, \phi^{\circ}$ is an isomorphism from $C\left(\mathbf{R}^{2}\right)$ onto a subspace of $C(\mathbf{R})$. It is thus enough to prove the corresponding statement between $L_{p^{-}}$ spaces. For all $f \in C\left(\mathbf{R}^{2}\right) \cap L_{p}\left(\mathbb{R}^{2}\right)$

$$
\int_{\mathbf{Z}}|f \circ \phi|^{p}=\sum_{n \in Z} \int_{\pi}^{n+1}|f \circ \phi|^{p}=\sum_{n \in Z} \int_{0}^{1}\left|f \circ \tau_{n}^{(2)} \circ r_{n} \circ \varphi_{n}\right|^{p}
$$

where, for all $n, \hat{\varphi}_{n}$ equals $\varphi$ or $\psi$. According to the definition of $\psi$ we can find positive constants $c_{1}$ and $c_{2}$ such that

$$
c_{1} \int_{I^{2}}\left|f \circ \tau_{n}^{(2)} \circ r_{n}\right| p \leq \int_{0}^{1}\left|f \circ \tau_{n}^{(2)} \circ r_{n}^{\circ} \circ \varphi_{n}\right|^{p} \leq c_{2} \int_{I^{2}}\left|f \circ \tau_{n}^{(2)} \circ r_{n}\right|^{p}
$$

for all $f$ and $n$. Since $r_{n}$ is an isometry, we can further write

$$
\left.\sum_{n \in Z} \int_{I^{2}}\left|f \circ \tau_{n}^{(2)}{ }_{n} r_{n}^{p}=\sum_{n \in Z} \int_{i^{2}}\right| f \circ \tau_{n}^{(2)}\right|^{p}=\sum_{n \in Z} \int_{Q_{n}}|f|^{p}=\int_{\mathbf{R}^{2}}|f|^{p} .
$$

Combining (39), (40) and (41) we see that $\phi^{\circ}$ is also an isomorphism from $L_{p}\left(\mathbb{R}^{2}\right)$ onto a subspace of $L_{p}(\mathbb{R})$.

\section{NOTE ADDED IN PROOF}

After the paper "A continuous surjection from the unit interval onto the unit square" and the reference [T] in it, "Averaging operators on spaces of continuous functions" were submitted, I realized that some of the results of $[\mathrm{T}]$ were already proved by $\mathrm{B}$. Hoffmann in "An injective characterization of Peano spaces", Topol. and Appl. 11 (1980), 37-46. This is why [T] does not appear anywhere. In this note we give the missing details of the proofs of Lemma 1 and Theorem 2 of "A continuous surjection from the unit interval onto the unit square".

Proof of Lemma 1: The map $\gamma: \Delta^{4} \rightarrow \Delta^{4}$ is continuous, since the 
first $m$ columns of $\gamma(A), A \in \Delta^{4}$ depend only on the first $2 m$ columns of $A$. We show that $\gamma$ is a surjection having a continuous right inverse. Let $D=\left(D_{1}, D_{2}, \ldots\right) \in \Delta^{4}$, where each $D_{m} \in \Delta_{1}^{4}$. We define the element $A \in \Delta^{4}$, using the same notation as in the definition of $\gamma$, as follows. Let $B_{1}=C_{1}$ $=D_{1}$. For $m>1$ we set $C_{m}=D_{m}$ and for $B_{m}$ we choose a matrix which is not equal to anyone of the matrices $D_{m}, D_{m}^{-}, \overline{0}$ or $\overline{1}$. We set $A=\left(A_{1}, A_{2}, \ldots\right)$, where $A_{m}=\left(B_{m}, C_{m}\right)$ for all $m$. It follows now directly from $1^{\underline{0}}$ in the definition of $\gamma$ that $\gamma(A)=D$. Moreover, the element $A$ depends continuously on $D$, since the first $2 m$ columns of $A$ depend only on the first $m$ columns of $D$.

We denote by $\gamma^{-1}$ the continuous right inverse of $\gamma$ constructed above.

Finally, we show that $\varphi$ is a surjection. Since $\bar{\sigma}$ and $\bar{\psi}$ are surjections, it is enough to prove that $\gamma \circ \eta \circ \mathrm{g}$ is surjective. Let $D \in \Delta^{4}$ be arbitrary and let $A=\left(A_{1}, A_{2}, \ldots\right)=\gamma^{-1}(D)$. Each $A_{m}, m>1$, contains both numbers 0 and 1 . Hence, $\eta^{-1}(A)$ is of the form $\left(\varepsilon_{m}\right)_{m=1}^{\infty}$, where both numbers 0 and 1 occur as $\varepsilon_{m}$ for arbitrarily large $m$. But for such sequences we have

$$
Q\left(\sum_{m=1}^{\infty} \varepsilon_{m} 2^{-m}\right)=\left(\varepsilon_{m}\right)_{m=1}^{\infty}
$$

so that $\gamma \circ \eta \circ Q$ is surjective.

Proof of Theorem 2. The original proof of Milutin's lemma, which is also presented in [LT], Proposition 2.4.21, shows that the continuous map $\sigma \circ(\psi, \psi): \Delta^{2} \rightarrow I$ admits a regular averaging operator. Hence, the same is also true for

$$
\bar{\sigma} \circ \bar{\Psi}: \Delta^{4} \rightarrow I^{2}
$$

Let $P,\|P\|=1$, be a projection from $C\left(\Delta^{4}\right)$ onto $(\bar{\sigma} \circ \bar{\psi}){ }^{\circ}\left(C\left(I^{2}\right)\right)$.

Let $\gamma: \Delta^{4} \rightarrow \Delta^{4}$ be as above. The operator $f \nrightarrow \gamma^{\circ} P\left(\gamma^{-1}\right)^{\circ} f$ is a contractive projection from $C\left(\Delta^{4}\right)$ onto $(\sigma \circ \bar{\psi} \circ \gamma){ }^{\circ}\left(C\left(I^{2}\right)\right)$. Hence, also $(\bar{\sigma} \circ \bar{\psi} \circ \gamma \circ \eta)^{\circ}\left(C\left(I^{2}\right)\right.$ is a 1 -complemented subspace of $C(\Delta)$. 
Let $\mathrm{Q}: I \rightarrow \Delta$ be the discontinuous map defined in (3). By (4), $\mathrm{Q}^{\circ}$ is an isometry from $C(\Delta)$ onto $D(I)$ so that there exists a contractive projection $R$ from $D(I)$ onto $\varphi^{\circ}\left(C\left(I^{2}\right)\right)$, where

$$
\varphi:=\sigma \circ \bar{\psi} \circ \gamma \circ \eta \circ \mathbf{e} .
$$

By Lemma $1, \varphi$ is continuous so that $\varphi^{\circ}\left(C\left(I^{2}\right)\right)$ is a subspace of $C(I) \subset D(I)$. The restriction of $R$ to $C(I)$ gives the desired projection.

\section{References}

[BT] BONET, J. and TASKINEN, J. Non-distinguished Fréchet function spaces. Bull. Soc. Roy. Sci. Liége 58, 6 (1989), 483-490.

[LT] LINDENSTRAUSS, J. and TZAFRIRI, L. Classical Banach spaces. Springer Lecture Notes in Mathematics, 338.

[MT] MATTILA, P. and TASKINEN, J. Remarks on bases in a Fréchet function space, Rev. Mat. Univ, Complut., 6, N 1 (1993), 83-99.

[P] PEŁCZYŃSKI, A. Linear extensions, linear averaging and application to linear topological classification of spaces of continuous functions. Rozprawy Matematyczne 58 (1968).

[R] RUDIN, W. Real and complex analysis. Second edition. Mc Graw-Hill.

[T] TASKINEN, J. Averaging operators on spaces of continuous functions. Manuscript (1992).

Department of Mathematics

University of Helsinki

Hailituskatu 15

SF-00100 Helsinki

FINLAND
Recibido: 28 de agosto de 1992

Revisado: 10 de diciembre de 1992 